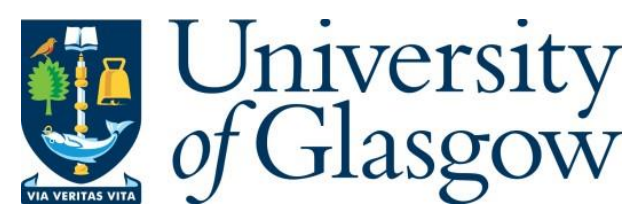

Sharma, P., Lao, L. and Falcone, G. (2018) A microwave cavity resonator sensor for water-in-oil measurements. Sensors and Actuators B: Chemical, 262, pp. 200-210.

There may be differences between this version and the published version. You are advised to consult the publisher's version if you wish to cite from it.

http://eprints.gla.ac.uk/165747/

Deposited on: 27 May 2020

Enlighten - Research publications by members of the University of Glasgow http://eprints.gla.ac.uk 


\title{
A Microwave Cavity Resonator Sensor for Water-In-Oil Measurements
}

\author{
Prafull Sharma, Liyun Lao and Gioia Falcone \\ Oil and Gas Engineering Centre, Cranfield University, United Kingdom
}

\begin{abstract}
Online monitoring of Water-Liquid Ratio (WLR) in multiphase flow is key in petroleum production, processing and transportation. The usual practice in the field is to manually collect offline samples for laboratory analysis, which delays data availability and prevents real time intervention and optimization. A highly accurate and robust sensing method is needed for online measurements in the lower end of WLR range (0\%-5\%), especially for fiscal metering and custody transfer of crude oil, as well as to ensure adequate flow assurance prevention and remedial solutions. This requires a highly sensitive sensing principle along with a highly precise measurement instrument, packaged together in a sufficiently robust manner for use in the field. In this paper, a new sensing principle is proposed, based on the open-ended microwave cavity resonator and near wall surface perturbation, for non-intrusive measurement of WLR. In the proposed concept, the electromagnetic fringe field of a cylindrical cavity resonator is used to probe the liquid near the pipe wall. Two of the cylindrical cavity resonance modes, TM010 and TM011 are energized for measurements and the shift in the resonance frequency is used to estimate liquid permittivity and the WLR. Electromagnetic simulations in the microwave frequency range of $4 \mathrm{GHz}$ to $7 \mathrm{GHz}$ are used for proof-of-concept and sensitivity studies. A sensor prototype is fabricated and its functionality demonstrated with flowing oil-water mixtures in the WLR range of $0-5 \%$. The frequency range of the proposed sensors is $4.4-4.6 \mathrm{GHz}$ and $6.1-6.6 \mathrm{GHz}$ for modes TM010 and TM011, respectively. The TM011 mode shows much higher sensitivity (41.6 MHz/WLR) than the TM010 mode (3.8 MHz/WLR). The proposed sensor consists of a $20 \mathrm{~mm}$ high cylinder, with a diameter of $30 \mathrm{~mm}$ and Poly-Ether-Ether-Ketone (PEEK) filler. The non-intrusiveness of the sensor, along with the high sensitivity in the resonance shift, makes it attractive for practical applications.
\end{abstract}

Keywords-Microwave resonator sensor, Water-Liquid Ratio, Water cut, Multiphase Flow Measurement

\section{Highlights}

- Proof-of-concept of a proposed sensing principle based on surface perturbation of an open-ended microwave cavity resonator.

- The fringe fields of the resonator are used to interact with the liquid medium enabling non-intrusive measurements.

- The TM011 mode is observed to be much more sensitive than the TM010 mode by a factor of nearly 11 .

\section{Introduction}

Monitoring of the WLR in multiphase flows is essential in petroleum production, processing and transportation. Traditionally, offline samples are taken in the field and used to measure the WLR in fluid analysis laboratories, making the data availability a lengthy process, with lack of real-time information. This, in turn, delays operational decision in the field, which may lead to unoptimized processes and lost production. A precise online measurement of WLR is highly desirable, but has proven to be challenging in the lower end of WLR range, particularly when high accuracy is required [1]. Online WLR meters (sometimes referred to as water cut meters) are commonly based on sensing one or combination of the dielectric permittivity, density, infrared or gamma-ray spectral absorption characteristics of the oil and water mixture [1].

Among these methods, WLR sensing using the contrast in the permittivity of oil and water has already been the subject of extensive academic as well as industrial research. One of the earliest proposals was made by Dykesteen et al. [2] consisting of a method of non-intrusive measurement of fluid fractions using two insulated electrodes and flowing multiphase mixture between them. The electrical impedance is measured and used to estimate the fractions of water, oil and gas. Methods based on electrical capacitance have also been developed, though measurement uncertainty and instrument drift issues limits their usage for high accuracy requirements. Hammer et al. [3] demonstrated a helically shaped capacitance electrode system for water fraction in oil-water mixtures with repeatability better than $1.5 \%$ [3]. They also demonstrated that helically shaped electrodes are less dependent on variation in flow regimes. Sami et al. [4] investigated a variety of capacitance designs to determine volume fractions in two-phase pipelines and concluded that a double helix electrode provides the most practical and linear sensor response in various flow regimes. Demori et al. [5] proposed a solution to the problem of capacitive sensing in the presence of conductive water, which introduces parasitic coupling to stray elements outside the measurement section of the pipe. They proposed a novel sensor configuration that employs guard electrodes, coupled to a tailored electronic interface to drive the guard electrodes. Chen et al. [6] investigated a coaxial capacitance sensor which they validated in a vertical upward oil-water two-phase flow experiment, and effectively applied time-frequency analysis to estimate water fraction in low water-cuts.

Electrical tomography based methods have been gaining interest especially for multiphase flow imaging and phase fraction estimation, although industrial versions for field use are still being developed. Yang [7] extensively reviewed the electrical capacitance tomography technology and provided design guidance. The measurement problem is particularly 
complex at the lower end of the WLR range $(0-5 \%)$, where poor contrast in dielectric permittivity $\left(\varepsilon_{\mathrm{r}}\right)$ between the oilwater mixture and the oil alone proves to be a challenge for applications demanding high accuracy [8].

Thanks to the increasing availability of precise electronics, driven by rapid developments in the telecom industry, microwave-based sensing solutions for robust and accurate WLR measurement have been emerging rapidly. Castle et al. [9] developed one of the earliest microwave-based sensing systems for water fraction measurement in crude oil applications. Microwave-based sensing principles can be broadly categorized into methods based on wave transmission, reflection and resonance. Various microwave sensing methods for industrial applications have been systematically reviewed by Nyfors [10].

Microwave resonance based principles are attractive for field use due to high precision and robustness against instrument drift [10]. Microwave based intrusive resonance sensor have been successfully demonstrated with real oil field applications and offered commercially [11]. They have also been used to estimate conductivity and salinity of water present in the multiphase mixtures [12]. Nyfors [13] developed an intrusive resonator method for detecting phase fraction in multiphase flows, particularly suited for wet gas applications. It consisted of a fin resonator as an intrusive feature of the pipe to create a resonator within the pipe structure. Subsequently, several other sensing principles were developed with non-intrusive microwave resonators. Wylie et al. [14] demonstrated a cavity resonator sensor principle which is nonintrusive and transmits low power $(10 \mathrm{~mW})$ radio frequency in the range of 100-350 MHz. They further developed an industrial version of the sensor and tested it in an industrial flow setting. Karimi et al. [15, 19] developed a planar microwave sensor with a T-resonator design for non-intrusive WLR sensing over the full range of operation $(0 \%-100 \%$ WLR). The proposed method uses screen printing with a 3D printed mask directly onto the pipe surface. The resonance frequency of the T-resonator changes in the frequency band of $90 \mathrm{MHz}-190 \mathrm{MHz}(111 \%)$ with changing water fraction in oil from $0 \%$ to $100 \%$.

Surface perturbation methods have gained research attention due the near wall measurement they provide that can be effective in measuring liquid properties even in the presence of gas. Furthermore, resonance based microwave sensors are evaluated for demanding applications, such as waste water with very low concentration of oil [16]. Ni and Ni [17] described a class of non-intrusive microwave resonator sensors, called extra-cavity perturbation methods, for generic applications. A sensing principle based on surface perturbation of a microwave dielectric resonator was developed by Nyfors [18] for application to wet gas flow with non-intrusiveness as a key advantage.

Zarifi et al. [20] established a microwave planar ring resonator sensor tuned at $5.25 \mathrm{GHz}$, providing a non-contact method for liquid-liquid interface detection. They demonstrated the applicability of this sensor to interface detection in waterolive oil-ethanol samples representing a wide range of permittivity. The resonator's Quality Factor (Q-Factor) is also used in the estimation process, in addition to the resonance frequency, to increase the robustness of the sensor.

Microwave resonators have been investigated for demulsification of water in crude oil using single- and multi-mode resonators [21].

Oon et al. [22] investigated a cylindrical microwave resonator sensor to monitor two phase flow systems and the changes in the permittivity of the measured phases to differentiate between the volume fractions of air, water and oil. Microwaves in the range of $5-5.7 \mathrm{GHz}$ have been used to analyse a two-phase air-water and oil-water stratified flow in a pipeline, demonstrating the ability to detect a change in water fraction in full range of $0 \%-100 \%$.

Zarifi and Daneshmand [23] proposed a non-contact liquid sensor using an active, feedback loop assisted, planar, microstrip microwave resonator. The sensor has the ability to operate in a non-contact fashion within a distance of 0 to $8 \mathrm{~cm}$. The active loop technique is shown to increase the primary Q-Factor from 210 to 500,000 in air when measured at a resonant frequency of $1.52 \mathrm{GHz}$. The proposed device is used to distinguish between water, ethanol, methanol, isopropanol, and acetone in a submerged tube inside a water-filled container. They also demonstrated the application of micro-strip microwave resonator sensor in monitoring solid particle deposition in lossy medium [24].

Al-Kizwini et al. [25] proposed a non-intrusive sensor, which is based on an electromagnetic waves cavity resonator. It determines and monitors the percentage volumes of each of the two phases (oil and gas) in the pipeline using the resonant frequencies shifts that occur within the resonator. Temperature has a significant influence on the liquid permittivity, especially for water. It also affects the resonance frequency of the measurement by microwave resonators and hence it is important to compensate the measurements against variations due to temperature [26].

From the literature review, it can be observed that microwave resonator based sensing principles have been gaining more and more attention with varied multiphase flow measurement applications. In this paper, in order to address the need for online, near-wall WLR sensing, a novel, non-intrusive surface perturbation microwave cavity resonator is proposed in the frequency range of $4.4-4.6 \mathrm{GHz}$ and $6.1-6.6 \mathrm{GHz}$, with active modes TM010 and TM011, respectively. Measurement of low WLR in two-phase (oil-water) flow and the proof-of-concept is established by means of simulations and experiments at constant temperature.

\section{Sensing Principle}

A schematic of the proposed cylindrical cavity resonator sensor in contact with the flowing liquid is shown in Figure 1. The flat open surface of the sensor interacts with the liquid to measure its permittivity. A key feature of this sensor is the use of fringe fields of the resonator to probe the liquid flowing near the pipe wall. This aspect enables the sensor to be 
physically non-intrusive, while only the sensing electromagnetic (EM in the Figure) fringe fields penetrate the pipe.

The resonance frequency of the sensor shifts with a change in liquid permittivity, which is then used to estimate the WLR. Since the sensor only probes a small portion of the flowing mixture near the pipe wall, a homogeneously mixed liquid is desirable for accurate estimations.

\subsection{Cavity Perturbation Method}

In conventional methods using resonant cavity perturbation, the material to be characterized is placed at a predefined location within the electromagnetic field, usually well inside the resonator cavity. The permittivity of the material is then estimated from the shift of the resonant frequency and Q-Factor of the resonance using predefined models or calibration curves.

The perturbation measurement principle assumes that the overall electromagnetic field of a resonating cavity, with a test material occupying a small fraction of the resonator volume, is not greatly different from that of the unperturbed cavity. This assumption of a small perturbation induced by the test materials, when violated, may lead to resonance mode conversion to a different mode, or even vanishing of the resonance altogether. Such a condition may arise if the material occupies a significant fraction of the resonator cavity's volume and/or has a highly lossy permittivity (materials with high microwave absorption such as saline water). Hence the conventional cavity-perturbation method is ineffective in these cases. An alternative way to address this problem is to place the sample externally to the resonator cavity, but still coupled with the cavity through evanescent fields or fringe fields of the resonator. In this case, the perturbation to the cavity due to the test material can be small and the resonant perturbation requirements may still be fulfilled. These methods fall into the category of the so-called extra-cavity perturbation methods [17] and have the potential to operate in much wider permittivity range of the test medium than the conventional resonator perturbation methods.

Figure 2 shows an illustration of perturbation of a cavity.

As a generalization, the material filling a part of the cavity resonator is denoted by symbol $V$. The symbols $V_{c}$ and $V_{s}$ represents control volume and supplied volume respectively. $\mathbf{E}_{\mathbf{1}}$ and $\mathbf{H}_{\mathbf{1}}$ represent the electric field and magnetic field of the unperturbed cavity, respectively. The Maxwell's curl equations [17] deduce the following equation:

$$
\frac{\Delta \omega}{\omega}=-\frac{\int_{V_{c}}\left(\Delta \epsilon\left|\boldsymbol{E}_{1}\right|^{2}+\Delta \mu\left|\boldsymbol{H}_{1}\right|^{2}\right) d V}{\int_{V_{c}}\left(\epsilon\left|\boldsymbol{E}_{1}\right|^{2}+\mu\left|\boldsymbol{H}_{1}\right|^{2}\right) d V}
$$

Where $\omega$ and $\Delta \omega$, represents the resonance frequency of the unperturbed cavity and the shift in the resonance frequency in the perturbed cavity, respectively. The resonance cavity is perturbed by a change in dielectric permittivity $(\varepsilon)$ and/or magnetic permeability $(\mu)$. Equation 1 indicates that any increase in $\varepsilon$ or $\mu$ decreases the resonance frequency of the cavity. In this research, an open-ended cylindrical microwave cavity sensor is proposed. The cylindrical cavity resonator sensor is filled with a dielectric filling material. The resonator sensor is enclosed in a metallic cavity; one flat face of the cylinder is non-metallic to probe the liquid using fringing electromagnetic fields out of the resonator (Figure 3). A coaxial feed is used at the metallic flat end of the resonator to excite the resonator at microwave frequencies.

The coaxial feed is sequentially supplied with a sweep of microwave frequencies and the reflected signal from the feed is measured using a Vector Network Analyzer (VNA). The port voltage reflection coefficient, known as S11 in conventional theory [27], is lowest at the resonance frequency due to energy storage and dissipation by the resonator. Since the fringe fields at the open end of the resonator penetrate the liquid, the permittivity of the liquid affects the resonance frequency. By measuring the resonance frequency and using an empirical calibration equation, the permittivity of the liquid is estimated, which is then used in a mixture model to estimate the WLR.

\subsection{Design methodology of cavity resonator}

A conventional microwave cylindrical cavity resonator is in the form of a circular waveguide with metallic boundaries in the curved and flat ends. Analytical formulations for the estimation of resonance frequencies of such cylindrical metallic cavity are well established [27]. However, the proposed sensing principle needs the cavity to be filled with dielectric filler and one of the flat ends of the cylindrical cavity to be non-metallic, open to the test medium. An analytical formulation for resonance frequency of such type of geometry is not known to be available and hence, for reasonable design guidance, the closed-ends formulation is used for estimating the resonance frequency of the unperturbed cavity. Simulation and experimental studies are carried out to establish the actual resonance frequencies of the open-ended microwave cavity resonator.

An illustration for the enclosed cylindrical cavity resonator is given in Figure 4. Here, 'a' and ' $d$ ' are the radius and height of the cylindrical cavity, respectively. Inside the cavity resonator, the electric and the magnetic fields oscillate within the cavity, and the power is dissipated in the metallic walls as well as within the dielectric filling material. The dielectric filler also affects the resonant frequency and Q-value. 
There are two categories of modes in a cylindrical cavity: Transverse Electric ( $\left.\mathrm{TE}_{\mathrm{mnp}}\right)$ and Transverse $\mathrm{Magnetic}\left(\mathrm{TM}_{\mathrm{mnp}}\right)$, depending on whether the direction of the electric or the magnetic field has any vector component along the cylinder axis, respectively. The index 'mnp' indicates the modal nodes of the field patterns in the directions $r, \varphi$ and $\mathrm{z}$ in the cylindrical coordinate system. The resonant frequency is related to the dimensions of a closed cavity and the dielectric properties of the filler material by equations 2 and 3 [27].

$$
\left(f_{r}\right)_{T M_{m n p}}=\frac{1}{2 \pi \sqrt{\mu \varepsilon}} \sqrt{\left(\frac{P_{m n}}{a}\right)^{2}+\left(\frac{p \pi}{d}\right)^{2}}
$$

where $J_{\mathrm{m}}\left(P_{\mathrm{mn}}\right)=0$

$$
\left(f_{r}\right)_{T E_{m n p}}=\frac{1}{2 \pi \sqrt{\mu \varepsilon}} \sqrt{\left(\frac{P_{m n}^{\prime}}{a}\right)^{2}+\left(\frac{p \pi}{d}\right)^{2}}
$$

where: $\mathrm{J}_{\mathrm{m}}$ is the Bessel function of $\mathrm{m}^{\text {th }}$ order; $\mathrm{P}_{\mathrm{mn}}$ and $\mathrm{P}^{\prime}{ }_{\mathrm{mn}}$ are the $\mathrm{n}^{\text {th }}$ root of $\mathrm{J}_{\mathrm{m}}$ and the derivative, respectively; $J_{\mathrm{m}}^{\prime}\left(P{ }_{\mathrm{mn}}\right)=0$. The symbol ' $p$ ' denotes the third dimension of the mode index 'mnp'.

Referring to the notations used in Figure 4 , in the scenario when $2 \mathrm{~d}>2 \mathrm{a}>\mathrm{d}$, the two fundamental TM modes of the cylindrical cavity are TM010 and TM011. Figure 5 shows the pattern of the electric fields (solid lines) and magnetic fields (dashed lines) of the TM010 mode and TM011 mode in an enclosed cylindrical cavity resonator. It can be noted that the magnetic field is parallel to the cavity bottom and perpendicular to the electric field, a condition of the TM modes.

When the enclosed cavity is made non-metallic at the bottom flat boundary of the cylinder, the dielectric filler at the flat surface is exposed to the medium surrounding it. When this dielectric surface gets in contact with the liquid, the resonance frequency shifts in accordance with the liquid permittivity. The sensor's resonance frequency, $f_{r, \text { sensor }}$ is related to that of the enclosed cavity, $f_{r}$ and to the resonance shift $\Delta f$ as a function of mixture permittivity $\left(\varepsilon_{m}\right)$, according to equation 4 for $\mathrm{TE}_{\mathrm{mnp}}$ mode or equation 5 for $\mathrm{TM}_{\mathrm{mn}}$ mode:

$$
\begin{array}{ll}
\left(f_{r, \text { sensor }}\right)_{T E_{m n p}}=\left(f_{r}\right)_{T E_{m n p}}+\Delta f\left(\varepsilon_{m}\right)_{T E_{m n p}} & \text { Eq. } 4 \\
\left(f_{r, \text { sensor }}\right)_{T M_{m n p}}=\left(f_{r}\right)_{T M_{m n p}}+\Delta f\left(\varepsilon_{m}\right)_{T E_{m n p}} & \text { Eq. } 5
\end{array}
$$

\subsection{WLR Estimation Model}

The measured sensor's frequency shift from a reference frequency is used in an empirical transducer model to estimate the permittivity of the liquid mixture $\left(\varepsilon_{m}\right)$. Figure 6 shows the estimation workflow for a dielectric permittivity based sensor for oil-water mixtures. The water permittivity $\left(\varepsilon_{w}\right)$, oil permittivity $\left(\varepsilon_{o}\right)$ and mixture permittivity $\left(\varepsilon_{m}\right)$ are used in a mixture model to calculate WLR. Water permittivity can be calculated using the model developed by Stogryn [28] for known water temperature, salinity and the electrical frequency.

There are several mixture models that can be used to relate permittivity of water, oil, and oil-water mixture to WLR. A detailed description of effective medium mixture models is given by Sihvola [29]. A commonly used effective medium model for homogeneous oil-water mixtures is the Bruggeman model [29] and it is used in this work for estimating WLR. Since oil-water mixtures can exist as oil-continuous or water-continuous, two different forms of the Bruggeman model are shown for the two cases in equations 6 and 7, respectively. The two equations forms are shown below for sake of completeness of discussion, although the application of the developed sensor is aimed at low WLR application which very likely lies in the oil continuous mixture type.

$$
\begin{array}{cc}
\alpha_{w}=1-\frac{\varepsilon_{w}-\varepsilon_{m}}{\varepsilon_{w}-\epsilon_{o}}\left(\frac{\epsilon_{o}}{\epsilon_{m}}\right)^{\frac{1}{3}} & \text { Eq. } 6 \\
\alpha_{w}=\frac{\varepsilon_{o}-\varepsilon_{m}}{\varepsilon_{o}-\epsilon_{w}}\left(\frac{\epsilon_{w}}{\epsilon_{m}}\right)^{\frac{1}{3}} & \text { Eq. } 7
\end{array}
$$

The symbols $\alpha$ and $\varepsilon$ represent the volumetric fraction and permittivity respectively. The subscripts $o, w, m$ represents oil, water and oil-water mixture respectively

It should be noted that, although permittivity is a complex quantity, the imaginary component of permittivity is negligible due to the non-conductive properties of oils and also of oil-water mixtures at low WLR (which is the focus of this research). Figure 7 shows a representative plot of the permittivity as estimated using the mixture models shown in Equations 6 and 7. 
In reality, as the WLR increases, the permittivity as predicted by the oil-continuous curve can only be appropriate up to a transition zone, after which the water-continuous curve would apply. The transition zone is commonly known to exist anywhere between WLR 30-70\%, but it can also occur outside this range.

\section{Design and Simulation Study}

An axisymmetric electromagnetic simulation model was developed using CST Microwave Studio version 2015 and a Finite Domain Time Domain (FDTD) solver was used to simulate the sensor response. Simulations were also carried out to optimize the feed, visualize the electric fields at resonance and validate the resonance shift with the permittivity of the liquid under test. For each test case, the port voltage reflection coefficient S11 was recorded. The minima of the S11 spectral curve represent resonance conditions due to increased stored energy and dissipation at the resonance.

The specific dimensions of the resonator sensor were chosen in consideration of the designed operational frequency and to physically fit into a pipe of nominal diameter $50 \mathrm{~mm}$. The dimensions of the cavity are chosen to be $30 \mathrm{~mm}$ in diameter and $20 \mathrm{~mm}$ in height. The feed to the resonator is symmetric enabling practical manufacturing and installation of the sensor into a spool. The design shown in Figure 8 was simulated with excitation frequencies from $2 \mathrm{GHz}$ to $7 \mathrm{GHz}$ are applied to the coaxial feed and the resonance frequency is estimated from the minima of the S11 spectral curve.

The permittivity of the liquid medium, calculated using the Bruggeman model, is sequentially varied from 2.3 to 3.0 to represent WLR values from $0 \%$ to $9 \%$, as shown in Table 2 .

The oil permittivity is assumed to be 2.3 (at $4.5 \mathrm{GHz}$ without dispersion effects), which is in mid-range of 2-2.5 as observed by Kjetil et al. [30] for a variety of oils. Water permittivity is calculated to be 63.04 at $4.5 \mathrm{GHz}$ using Stogryn's model [28] and represents produced saline water with $4 \% \mathrm{NaCl}$ (by weight $\%$ ) at $26^{\circ} \mathrm{C}$.

Figure 9 shows the electric field intensity generated from simulations when the feed is excited at the resonance frequency of $4.45 \mathrm{GHz}$. It can be seen that, while most of the electromagnetic field is confined within the resonator cavity, there exist a fringe field outside the resonator, which penetrates the dielectric medium placed in contact with the bottom open end of the resonator. This fringe field helps to probe the liquid medium to measure its permittivity. Figure 10 shows the simulated electric field pattern at TM011 mode $(6.62 \mathrm{GHz})$, which, as expected, has a very different modal pattern than that of the TM010 mode. The TM011 mode also shows a fringing field pattern into the test medium.

For the sensor dielectric filling material, two commonly used, chemically inert materials representing a low and a high permittivity are studied: a) PEEK - a thermoplastic with $\varepsilon_{r}$ of 3.2, and b) ALUMINA - a ceramic with $\varepsilon_{r}$ of 9.8. The permittivity of the filling material is expected to have an impact on the sensitivity of the sensor and hence this particular aspect was investigated.

The simulations confirmed the energization of the TM010 and TM011 modes, as can be seen in Figures 9 and 10, as well as in Figure 11. It can be observed that, in Figure 11, with oil as the test material, the PEEK filler showed resonance of the TM010 and TM011 modes at $4.45 \mathrm{GHz}$ and $6.62 \mathrm{GHz}$, respectively. With ALUMINA, on the other hand, both these modes expectedly appeared at a lower frequency of $2.55 \mathrm{GHz}$ and $4.25 \mathrm{GHz}$, due to the higher permittivity value of ALUMINA.

Figure 12 and Figure 13 shows the response curves of the sensor with PEEK filler for the TM010 and TM011 modes respectively. It can be observed that, with an increase in the permittivity of the test medium, the resonance shifts to a lower value, thereby demonstrating proof-of-concept even at this low WLR.

Using simulations and electric field visual representation (Figure 9 and 10), it is observed that the electromagnetic fringe field penetrates the test liquid, thereby coupling the resonator with the test medium. A perturbation in the resonator due to test medium leads to a shift in the resonance frequency, as shown in Figure 12 and 13.

Figure 14 and Figure 15 compare the sensitivity of the TM010 mode to that of the TM011 mode for both cases with PEEK and ALUMINA as the fillers. With both dielectric fillers, the TM011 mode shows higher sensitivity than the TM010 mode. In the case of PEEK, the sensitivity of the TM010 mode is $3.4 \mathrm{MHz} / \mathrm{WLR}$ and that of the TM011 mode is $41.0 \mathrm{MHz} / \mathrm{WLR}$. In the case of ALUMINA, the sensitivity of the TM010 mode is $1.2 \mathrm{MHz} / \mathrm{WLR}$ and that of TM011 mode is $4.9 \mathrm{MHz} / \mathrm{WLR}$. The simulated measurement sensitivity with PEEK is observed to be higher than that of ALUMINA for both the TM010 and TM011 modes by a factor of 2.8 and 8.3 , respectively.

\section{Experiments with Oil-Oil Static Mixtures}

A prototype of the sensor was fabricated with the dimensions shown in Figure 8. The sensor body is made of stainless steel 316 and the dielectric filler is made of PEEK. Figure 16 shows a picture of the prototype.

To experimentally prove the sensor concept, a set of test liquids were required, which needed to exhibit a range of 
permittivity values between 2 to 3 and remain miscible for a few weeks for repeatability of the experiments. The oil-water mixtures were not ideas for this validation as under stationary conditions, they tend to separate quickly unless emulsifiers are used. Moreover, the use of emulsifier may alter the permittivity of the oil-water mixtures. Hence, test samples were prepared by blending two different oils of known permittivity to test the sensor in a static manner as oil-oil mixtures are much more stable and controllable. The two oils were mixed using a high-speed blender at $10000 \mathrm{rpm}$ for 5 minutes to produce homogenous, stable liquid samples of different mixture permittivity. One oil was EDM mineral oil, referred to as $\mathrm{Oil}_{1}$ in this paper, and the other was Castor oil (Brand name Holland and Barret), referred to as Oil ${ }_{2}$ in this paper, with measured permittivity of 2.07 and 2.66, respectively. After mixing, the samples were allowed to settle for over 24 hours, to allow trapped air bubbles to escape. The permittivity range of these samples is representative of typical produced field oil-water streams in the WLR range of $0-5 \%$.

Figure 17 shows a schematic of the experimental setup for validation of the sensor response and sensitivity in a controlled static condition. The sensor is shown to be in contact with a carefully prepared sample of oil-oil homogenous mixtures. The applied signals and reflected signals are measured using 1-port Vector Network Analyzer (VNA) R140 Copper Mountain and the data is stored on a PC.

Each sample of liquid was characterized for dielectric permittivity using SPEAG DAK3.5 Coaxial probe [23] with an uncertainty of $+/-2.5 \%$ of permittivity value. The measured permittivity of the two oils is as shown in Figure 18 . Each sample was tested three times as indicated by Tiral1, Trial2 and Tiral3 in Figure 18 and 19. Oil 2 permittivity was found to have a frequency dependency possibly due to the complex molecular structure of vegetable castor oil. Five test mixture samples were prepared with equally spaced fraction from $0 \%$ to $100 \%$.

The permittivity of the test samples as measured by the dielectric probe kit is as shown in Figure 19. The permittivity of the $\mathrm{Oil}_{1}-\mathrm{Oil} l_{2}$ mixture is representative of the permittivity of oil-water mixtures at WLR $<5 \%$.

Figure 20 shows the response curves of the simulation and experiments. It can be observed that there are two resonances in both simulations and experiments. Since, analysis of simulation electric field patterns attributed the two resonances to be TM010 and TM011 modes, the experimentally observed resonances are also attributed to these two modes in this paper. Also, although there are two resonances observed between $3 \mathrm{GHz}$ till $7 \mathrm{GHz}$, a slight difference between simulation and experiments can be attributed to the difference in the oil permittivity, as well as the actual permittivity of the PEEK filler.

It is expected that the resonance should shift to a lower value when the sensor is taken from air to mineral oil. Figure 21, shows that both the TM010 mode and TM011 modes shift to a lower value with oil, and that the TM011 resonance shifts much more than for the TM 010 mode. The resonance quality of the TM011 mode is affected considerably when oil is introduced.

\section{Experiments with Water-In-Oil Flowing Mixtures}

Experimental tests of the sensor were performed in a flow test rig with homogenous oil-water mixtures at various WLR\% with a liquid flow rate of 1.0 litre per second (flow speed of 0.5 meters per second) in a $50 \mathrm{~mm}$ ( 2 -inch) nominal pipe diameter. The chosen flow rate and flow mixers produced a homogenous flowing mixture. This is because, if the liquid is not uniformly mixed, this may lead to a significant deviation from the mixture model (equation 6) used for permittivity estimation from water fraction, in which homogeneity is a key assumption [29]. Figure 23 shows a schematic of the experimental setup assembled for validation of the sensor response and sensitivity. The sensor was installed in the spool housing made of stainless steel. The spool was installed on a vertical PVC pipe 36D downstream of the bottom bend of the pipe.

The flow setup contains a liquid tank of 16 litres capacity which is manually prefilled with a pre-calculated known mass of oil and water. The liquid pump used is Cole-Parmer positive displacement impeller pump 5.3A. There are liquid sampling taps placed just before and after the pump. A static liquid mixer is placed downstream of the pump to mix the oil-water mixture as it enters the vertical PVC pipe.

Mineral oil used in the setup is Shell Tellus 46 with a measured permittivity of 2.07. The water used is saline water with $4 \%$ by weight of $\mathrm{NaCl}$ in water to represent nominal produced water salinity.

The temperature of the liquid was controlled and set to $26 \mathrm{DegC}+/-0.1^{\circ}$ Celcius using a Cole-Parmer Polystat $6 \mathrm{~L}$ Chiller/Heater re-circulator.

The sensor is connected to Vector Network Analyser (VNA), Copper Mountain RVNA140, and a PC to drive the VNA and store the acquired data. A frequency sweep was performed from $4 \mathrm{GHz}$ to $8 \mathrm{GHz}$ in steps of $1 \mathrm{MHz}$ at the rate of $2 \mathrm{~s} /$ frequency sweep. A total of 15 sweeps were acquired for each WLR test point and averaged to report the results.

The WLR $\%$ was varied from $0 \%$ to $5 \%$ in steps of $1 \%$. The Bruggeman model, as shown in equation 6 , is used to relate the reference WLR\% with reference permittivity of the test mixture (Table 3).

The reflection parameter S11 of the sensor with different WLR points is shown in Figure 25 for TM010 mode and Figure 
26 for TM011 mode. It can be observed that the resonance frequency (frequency at the minima of response curve) shifts to a lower value with increase in the WLR. This monotonic shift of resonance frequency demonstrates the conceptual working of the sensor for the intended application. The resonance curves of TM011 mode were broad and with undulations indicating that this mode might be more dissipative than the TM010 mode. Hence automated tracking of resonance is more difficult in TM011 mode than in TM010 mode.

The experimental resonance shift (Figure 27) shows that TM011 mode has much higher sensitivity than TM010 mode. It can be seen that TM011 mode frequency shift in Figure 27 shows slight nonlinearity as compared to simulations (Figure 13) and static oil-oil experiments (Figure 22). This difference may be due to the fact that the experimental results of Figure 27 are obtained when the sensor is installed into the spool, and there are likely cylindrical waveguide modes of spool affecting the sensor resonance behaviour.

The experimental Q-Factor of the resonance curve of TM010 mode is shown in Figure 28. Since the resonator is an open ended type, the resonance curve shows a low Q-Factor shape possibly due to radiative losses and losses due to dielectric liquid in proximity. It can be observed from Figure 28 that Q-Factor monotonically decreases with an increase in WLR\% although in a highly non-linear fashion. This is challenging for measurement at low WLR, especially in the range $0 \%$ $2 \%$. Due to low Q-Factor, the resonance peak is not sharp which makes picking the resonance point difficult. This may be addressed by increasing the frequency resolution of the frequency sweep. In the current experiment, a frequency resolution of $1 \mathrm{MHz}$ is used in the measurement, which is observed to give reasonable trend in shift in Q-Factor.

A comparison of simulation and experimental sensitivity shows a close match both for TM010 as well as TM011 modes (Figure 30). It should be noted that the oil permittivity used in the simulation (2.3) is not same as that of oil used in flow experiments (2.07).

The sensitivity with respect to WLR for TM011 mode is observed to be $41.6 \mathrm{MHz} / \mathrm{WLR} \%$ and for TM010 mode 3.8 $\mathrm{MHz} / \mathrm{WLR} \%$ for a range of $0 \%-1 \% \mathrm{WLR} \%$.

The WLR estimation steps shown in Figure 6 were used to estimate WLR for the oil-water flow experiments. The flow rig reference uncertainty is $+/-0.1$ of absolute WLR $\%$. A set of points were run as calibration points to build empirical calibration models for both TM010 and TM011 modes, relating resonance shift to the mixture permittivity, estimated from WLR\% using Bruggeman model. Subsequently, the test points were set on the flow rig and using the calibration model, the measured resonance shift is used to estimate mixture permittivity and finally WLR\%.

A comparison of the estimated WLR\% with reference WLR\% is shown in Figure 30 for both the modes. The error in estimated WLR is shown in Figure 31 and the results are observed to be within a value of $+/-0.1$ absolute of reference WLR\%.

The temperature of the mixture in the experiments was kept constant at $26 \mathrm{DegC}$ using a chiller/heater re-circulator to eliminate the effect of temperature on permittivity and WLR estimation process. Usually, an increase in temperature significantly reduces the water permittivity [28] and will lead to a bias in WLR estimation if not compensated for. Hence the calibration of the sensor should ideally be performed at the temperature the sensor is intended to be used at.

\section{Discussions and Conclusions}

A proof-of-concept of a sensing principle based on surface perturbation of a microwave cavity dielectric resonator is demonstrated for non-intrusive low WLR measurement $(<5 \%)$. The shift in the modal resonance frequency is used to estimate test medium permittivity and subsequently the WLR\%. Effect of the dielectric filler permittivity is studied with two different materials. Two of the cylindrical cavity resonance modes, TM010 and TM011 are evaluated for sensitivity comparisons when the open face of the sensor is exposed to liquid medium. Electromagnetic simulations in the microwave frequency range are used for the sensor design, proof-of-concept and sensitivity studies. For the sensor dielectric filler, two commonly used chemically inert materials are studied representing different dielectric permittivity a) Poly-EtherEther-Ketone (PEEK) - a thermoplastic with $\varepsilon_{\mathrm{r}}$ of 3.2 and b) ALUMINA - a ceramic with $\varepsilon_{\mathrm{r}}$ of 9.8 .

Simulations confirmed the energization of TM010 and TM011 modes in the resonator sensor using electric fields analysis and frequency response curves.

PEEK filler showed resonance of TM010 and TM011 modes at $4.45 \mathrm{GHz}$ and $6.62 \mathrm{GHz}$ respectively. With ALUMINA, owing to a higher dielectric permittivity, the corresponding resonances were comparatively at lower frequency $2.55 \mathrm{GHz}$ and $4.25 \mathrm{GHz}$.

Using simulations and electric field visualization, it is observed that the electromagnetic fringe field penetrates the test liquid thereby coupling the resonator with test medium. A perturbation in the resonator due to test medium leads to a shift in the resonance frequency establishing the working of the sensing concept.

The simulations study revealed that with PEEK filler, sensitivity is observed to be higher than that with ALUMINA filler for both modes TM010 and TM011 by a factor of 2.8 and 8.3 respectively.

For both dielectric filler materials, TM011 mode showed higher sensitivity than TM010 mode. In the case of PEEK filler, TM010 mode sensitivity is $3.4 \mathrm{MHz} / \mathrm{WLR} \%$ and the sensitivity for TM011 mode is $41.0 \mathrm{MHz} / \mathrm{WLR} \%$. It showed that lower permittivity of dielectric filler material is preferable as they provide higher measurement sensitivity. 
A sensor prototype is fabricated and experimentally tested. Experimental validations are performed with controlled static mixtures of various permittivity created using two different miscible oils confirming the trends observed in simulation. Further experimental validation with flowing water-in-oil mixtures in the WLR range of $0 \%-5 \%$ is performed in a flow rig and the sensor sensitivity for both modes TM010 and TM011 are reported.

The experimental observations, like in simulation, showed that TM011 mode has a much higher sensitivity (41.6 $\mathrm{MHz} / \mathrm{WLR} \%$ ) than TM010 mode (3.8 MHz/WLR\%).

The resonance curves of TM011 mode were not sharp, were broad and with undulations indicating that this mode is more dissipative than the TM010 mode. Hence automated tracking of resonance is more difficult in TM011 mode than in TM010 mode.

The error in estimated WLR is observed to be within a value of $+/-0.1$ absolute of the reference WLR\%.

Overall, the demonstrated sensing concept showed feasibility for practical applications in lower end range of WLR\% $(0 \%-5 \%)$.

\section{Acknowledgements}

This research program is funded and supported by the Oil and Gas Engineering Center at Cranfield University. The authors are thankful to M-Flow Technologies Ltd and their staff, including Dr Alan Parker, Edward Giles and Dr Sahar Zamani, for allowing their flow test rig to be used in this work. The authors would like to thank Professor Emeritus Hoi Yeung for initiating this research.

\section{Nomenclature}

\begin{tabular}{ll}
\hline Symbol & Definition \\
\hline WLR & Water-Liquid Ratio \\
$\boldsymbol{\varepsilon}$ & Dielectric Permittivity \\
$\mu$ & Magnetic Permeability \\
$\boldsymbol{E}_{\boldsymbol{1}}$ & Electric field in unperturbed cavity \\
$\boldsymbol{H}_{\boldsymbol{1}}$ & Magnetic field in unperturbed cavity \\
$\omega$ & Resonance frequency (radians/s) \\
$V$ & Volume \\
$\mathrm{TM}$ & Transverse Electric mode \\
$\mathrm{TE}$ & Transverse Magnetic mode \\
$f$ & Resonance frequency (Hz) \\
$J_{m}$ & Bessel function of mth order \\
$P_{m n}$ & Root of $\mathrm{J}_{\mathrm{m}}\left(\mathrm{n}^{\text {th }}\right.$ root) \\
$\alpha_{w}$ & Fraction of water \\
\hline
\end{tabular}

\section{References}

[1] G. Falcone, G. Hewitt, C. Alimonti, Multiphase flow metering: principles and applications, vol. 54, Elsevier, 2009.

[2] E. Dykesteen, A. Hallanger, E.A. Hammer, E. Samnøy, R. Thorn, Non-intrusive three-component ratio measurement using an impedance sensor, J. Phys. E: Sci. Instrum. vol. 18 (1985) 540-544.

[3] E.A. Hammer, J. Tollefsen, K. Olsvik, Capacitance transducers for non-intrusive measurement of water in crude oil, Flow Measurement and Instrumentation. vol. 1, issue 1 (1989) 51-58.

[4] M.S.A. Abouelwafa, E.J.M. Kendall, The use of capacitance sensors for phase percentage determination in multiphase pipelines, IEEE Trans. Instrum. Meas. vol. 29, no. 1 (1980) 24-27.

[5] M. Demori, V. Ferrari, D. Strazza, P. Poesio, A capacitive sensor system for the analysis of two-phase flows of oil and conductive water, Sens. Actuators A, Phys. vol. 163, no. 1 (2010) 172-179.

[6] X. Chen, Y.F. Han, Y.Y. Ren, H.X. Zhang, H. Zhang, N.D. Jin, Water holdup measurement of oil-water two-phase flow with low velocity using a coaxial capacitance sensor, Experimental Thermal and Fluid Science. vol. 81 (2017) 244-255.

[7] W.Q. Yang, Design of electrical capacitance tomography sensors, Meas. Sci. Technol, vol. 21, Issue 4 (2010) 042001.

[8] I. Ismail, J.C. Gamio, S.F.A. Bukhari, W.Q. Yang, Tomography for multi-phase flow measurement in the oil industry, Flow Measurement and Instrumentation. vol. 16, issue 2 (2005) 145-155.

[9] G.S.P. Castle, J. Roberts, A microwave instrument for the continuous monitoring of the water content of crude oil, Proc. IEEE. vol. 62, no. 1 (1974) 103-108.

[10] E. Nyfors, P. Vainikainen, Industrial Microwave Sensors, first ed., Artech House, London, 1989.

[11] E.G. Nyfors, A. Wee, Measurement of mixtures of oil, water, and gas with microwave sensors: New developments and field experience of the MFI MultiPhase and WLR meters of Roxar, Proc. SPIE. vol. 4129 (2000) 12-21.

[12] O.L. Bo, E. Nyfors, Application of microwave spectroscopy for the detection of water fraction and water salinity in water/oil/gas pipe flow, Journal of Non-Crystalline Solids. vol. 305, Issue 1 (2002) 345-353.

[13] E. G. Nyfors, Cylindrical microwave resonator sensors for measuring materials under flow, PhD Dissertation, Dept. Elect. 
Commun. Eng., Helsinki Univ. Technol. Espoo, Finland, 2000.

[14] S.R. Wylie, A. Shaw, A.I. Al-Shamma, RF sensor for multiphase flow measurement through an oil pipeline, Meas. Sci. Technol. vol. 17, no. 8 (2006) 2141.

[15] M.A. Karimi, M. Arsalan, A. Shamim, Low Cost and Pipe Conformable Microwave-Based Water-Cut Sensor, IEEE Sensors Journal. vol. 16, no. 21 (2016) 7636-7645.

[16] O. Korostynska, A. Mason, A. Al-Shamma, Microwave sensors for the non-invasive monitoring of industrial and medical applications, Sensor Review, vol. 34 issue 2 (2014) 182-191.

[17] E.H. Ni, Y.Q. Ni, Extra-cavity perturbation method for the measurement of dielectric resonator materials, Review of Scientific Instruments, vol. 68, issue 6 (1997) 2524-2528.

[18] E. Nyfors, Flow Measurements, US Patent 8570050 B2, 2013.

[19] M.A. Karimi, M. Arsalan, A. Shamim, Dynamic characterization of a low cost microwave water-cut sensor in a flow loop, Sensors and Actuators A: Physical. 260 (2017) 146-152.

[20] M. H. Zarifi, M. Rahimi, M. Daneshmand, T. Thundat, Microwave ring resonator-based non-contact interface sensor for oil sands applications, Sensors and Actuators B: Chemical. 224 (2016) 632-639.

[21] D. Santos et al, Demulsification of water-in-crude oil emulsions using single mode and multimode microwave irradiation, Separation and Purification Technology. 189 (2017) 347-356.

[22] C.S. Oon et al, Experimental study on a feasibility of using electromagnetic wave cylindrical cavity sensor to monitor the percentage of water fraction in a two phase system, Sensors and Actuators A: Physical. 245 (2016) 140 -149.

[23] M. H. Zarifi, M. Daneshmand, Liquid sensing in aquatic environment using high quality planar microwave resonator, Sensors and Actuators B: Chemical. 225 (2016) 517-521.

[24] M. H. Zarifi, M. Rahimi, M. Daneshmand, Monitoring Solid Particle Deposition in Lossy Medium Using Planar Resonator Sensor, IEEE Sensors Journal. (2017).

[25] M.A. Al-Kizwini et al, The monitoring of the two phase flow-annular flow type regime using microwave sensor technique Measurement. 46.1 (2013) 45-51.

[26] S. Almuradi et al, Temperature Impact in Electromagnetic Non-Invasive Water/Oil/Gas Multiphase Real Time Monitoring, Asian Journal of Eng and Tech. vol. 3, issue 5 (2015) 512-527.

[27] D.M. Pozar, Microwave Engineering, fourth ed., John Wiley \& Sons, New York, 2011.

[28] A. Stogryn, Equations for Calculating the Dielectric Constant of Saline Water, IEEE Trans. Microwave Theory and Techniques, vol. 19, issue 8 (1971) 733-736.

[29] A. Sihvola, Electromagnetic Mixing Formulas and Applications, first ed., IET Publishing, London, 1999.

[30] F. Kjetil, A.L. Tomren, S. Froyen, Permittivity Calculator: Method and Tool for Calculating the Permittivity of Oils from PVT Data, 30th North Sea Flow Measurement Workshop, St. Andrews, (2012).

[31] SPEAG, DAK - High Precision Dielectric Measurements, Retrieved (August 27, 2017) from URL www.SPEAG.com/products/dak 


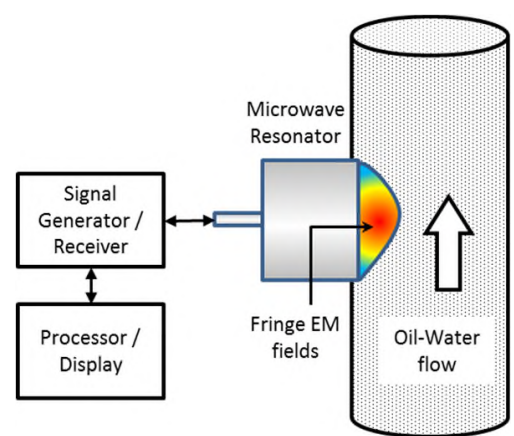

Figure 1: The fringe fields interactions of the microwave resonator sensor with oil-water flow
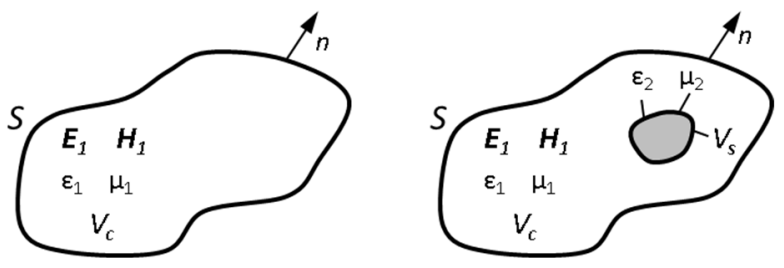

Figure 2: Cavity perturbation a) Unperturbed cavity b) Perturbed cavity

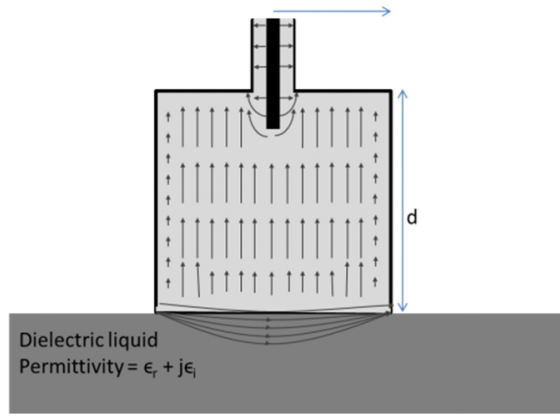

Figure 3: Sensing principle based on fringe fields of a cylindrical microwave resonator

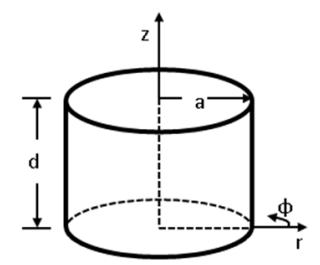

Figure 4: Cylindrical Cavity Resonator
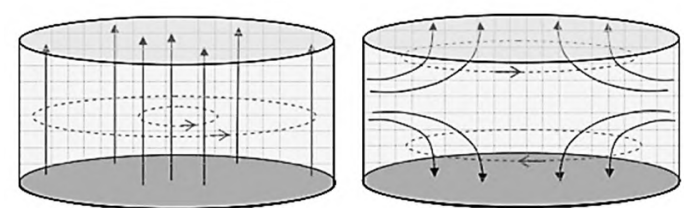

Figure 5: The Electric (solid lines) and Magnetic (dashed lines) Fields for a) TM010 mode b) TM011 mode 


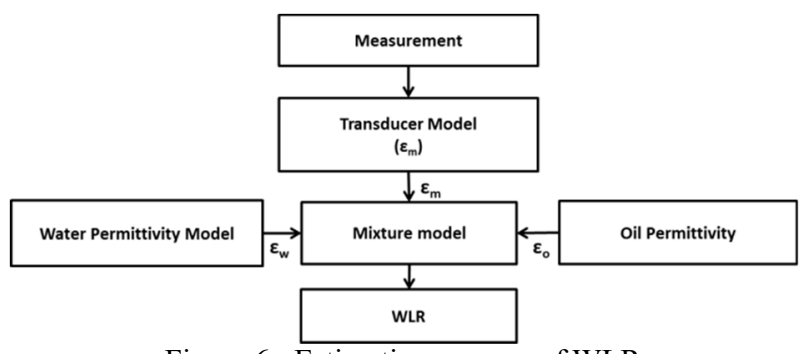

Figure 6: Estimation process of WLR

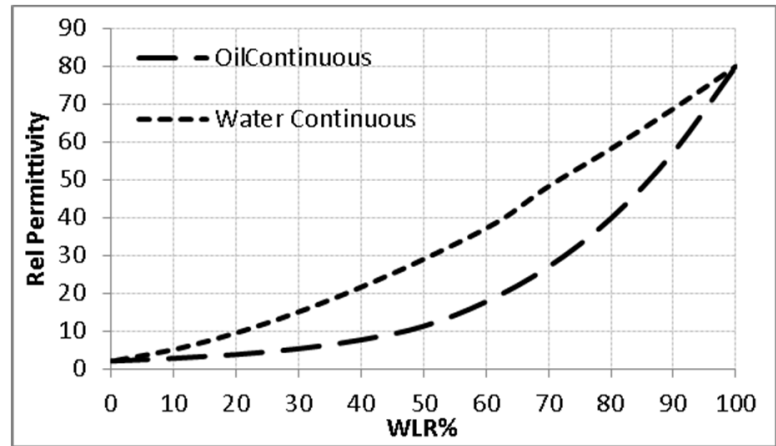

Figure 7: Plots representing the Bruggeman model to theoretically relate mixture permittivity from WLR\%

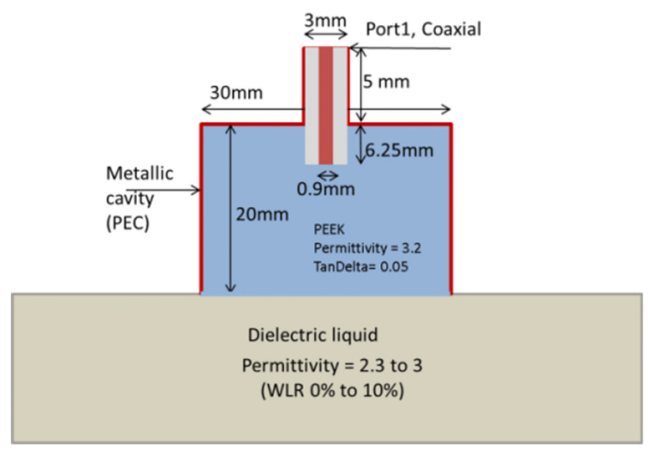

Figure 8: Dimensions of the axisymmetric simulation model

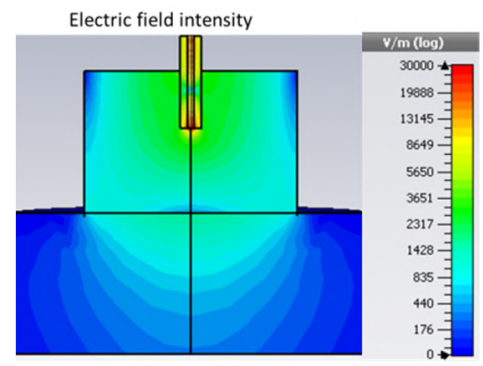

Figure 9: Simulated electrical field pattern at the TM010 mode resonance frequency $(4.45 \mathrm{GHz})$, with oil as test medium

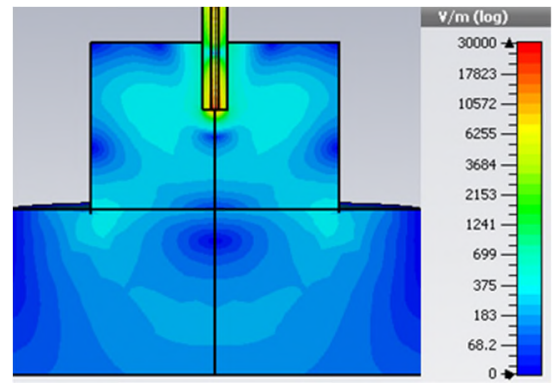

Figure 10: Simulated electrical field pattern at the TM011 mode resonance frequency $(6.62 \mathrm{GHz})$, with oil as test medium 


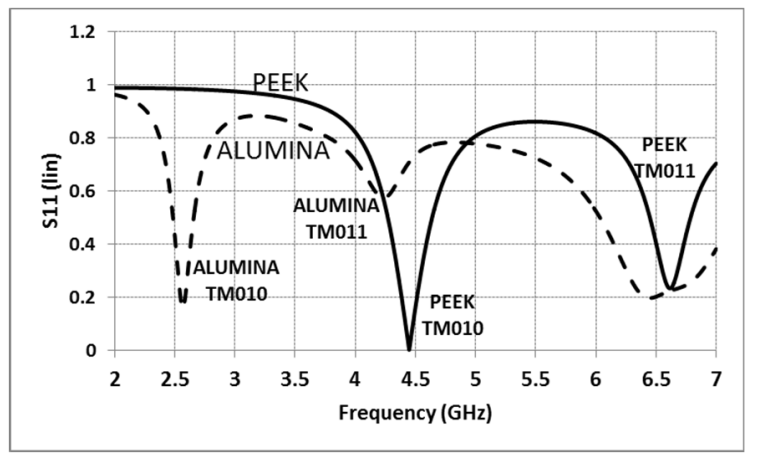

Figure 11: Simulated frequency response with oil medium (permittivity $=2.3$ )

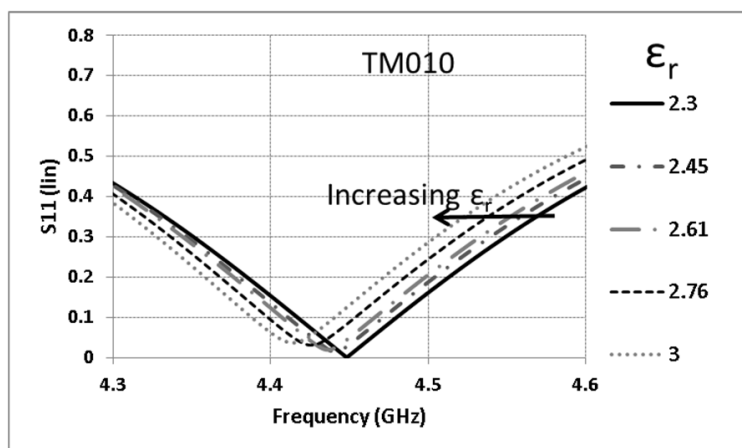

Figure 12: S11 response curves of TM010 mode with PEEK filler

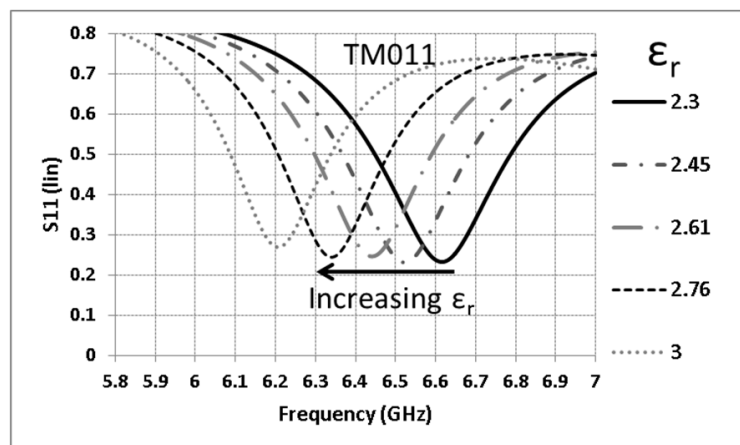

Figure 13: S11 response curves of TM011 mode with PEEK filler

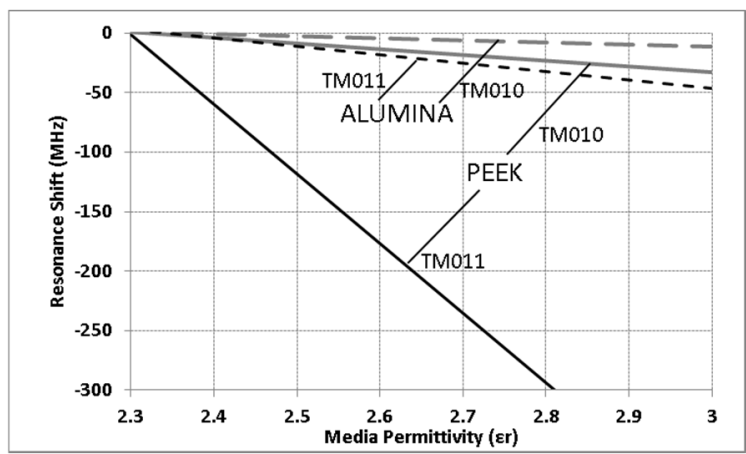

Figure 13: Simulated resonance frequency of various medium permittivity with resonator with a) PEEK filler b) ALUMINA filler 


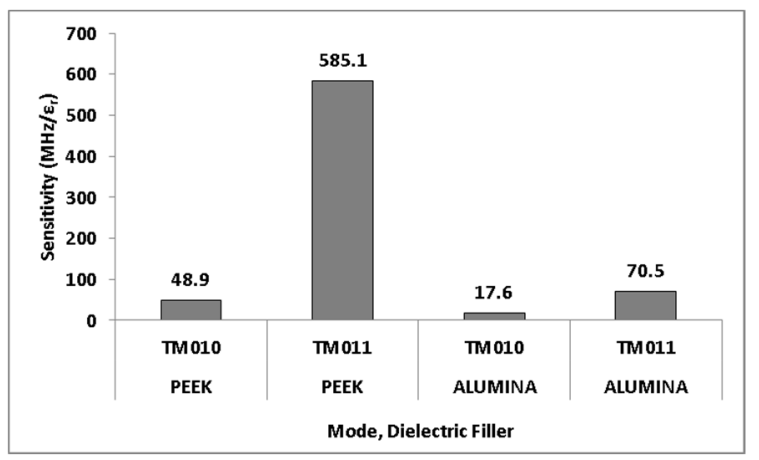

Figure 14: Sensitivity $\left(\mathrm{MHz} / \varepsilon_{\mathrm{r}}\right)$ of modes TM010 and TM011 with dielectric fillers as PEEK and ALUMINA

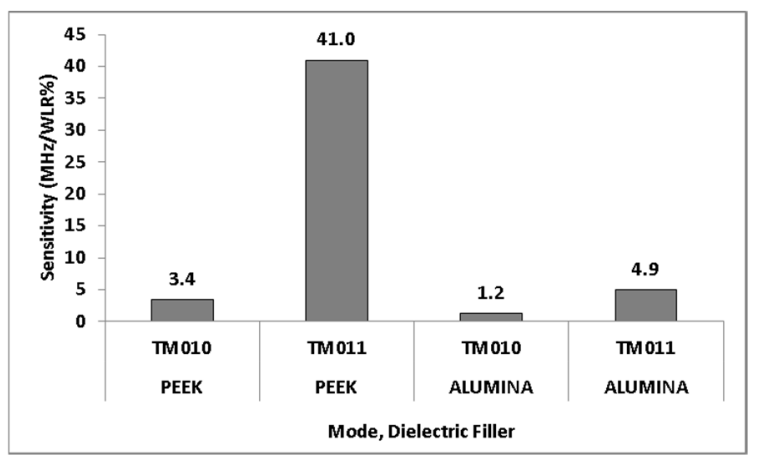

Figure 15: Sensitivity (MHz/WLR\%) of modes TM010 and TM011 with dielectric fillers as PEEK and ALUMINA

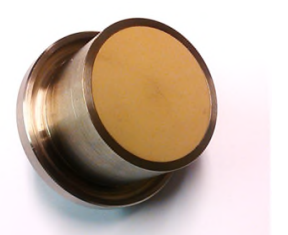

Figure 16: Sensor prototype with PEEK filler

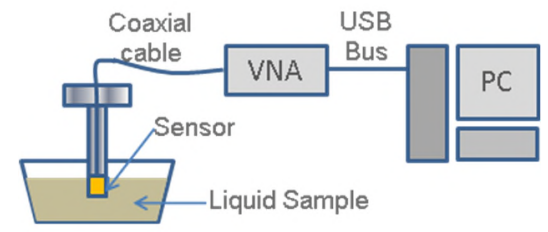

Figure 17: Experimental setup for sample preparation and measurement

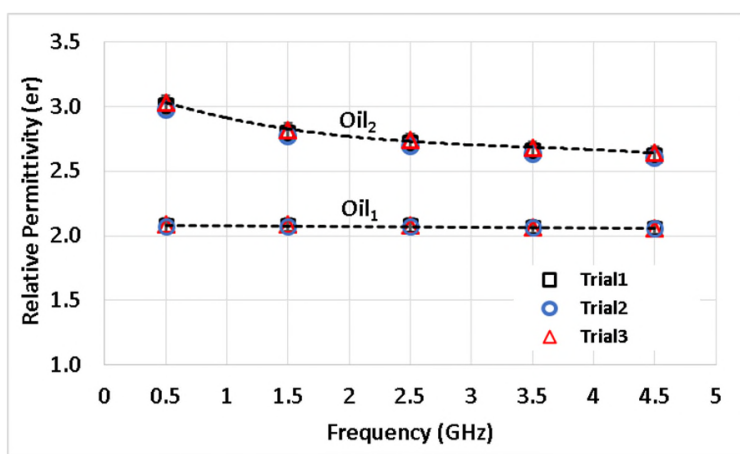

Figure 18: Permittivity characterization of $\mathrm{Oil}_{1}$ and $\mathrm{Oil}_{2}$ 


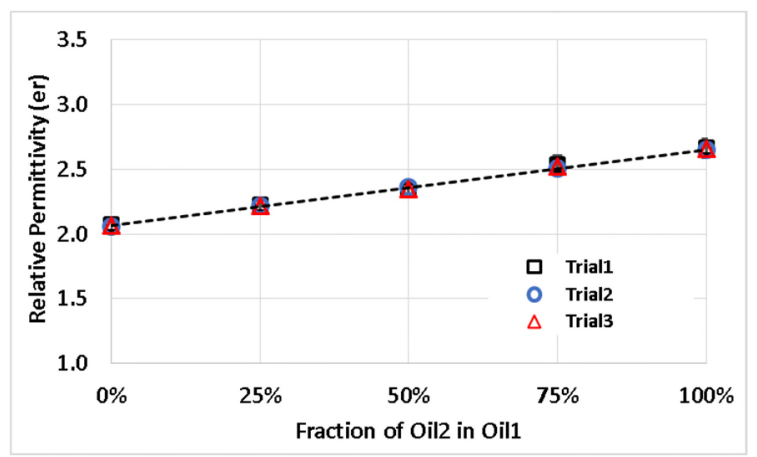

Figure 19: Permittivity of $\mathrm{Oil}_{1}-\mathrm{Oil}_{2}$ mixture at $4.5 \mathrm{GHz}$

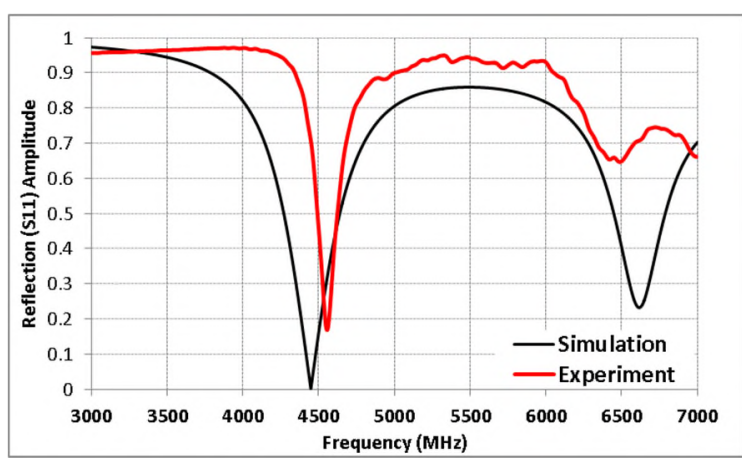

Figure 20: Simulation and Experimental response of resonator sensor with mineral oil

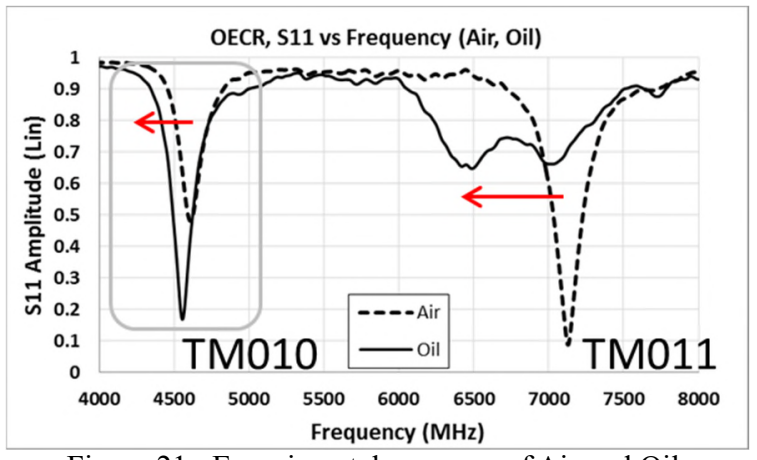

Figure 21: Experimental response of Air and Oil 1

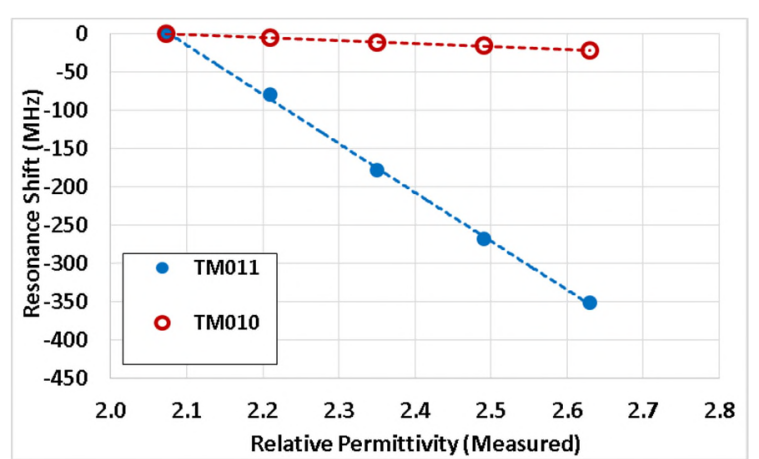

Figure 22: Experimental response sensitivity comparison of TM010 and TM011 modes in experimental oil mixtures 


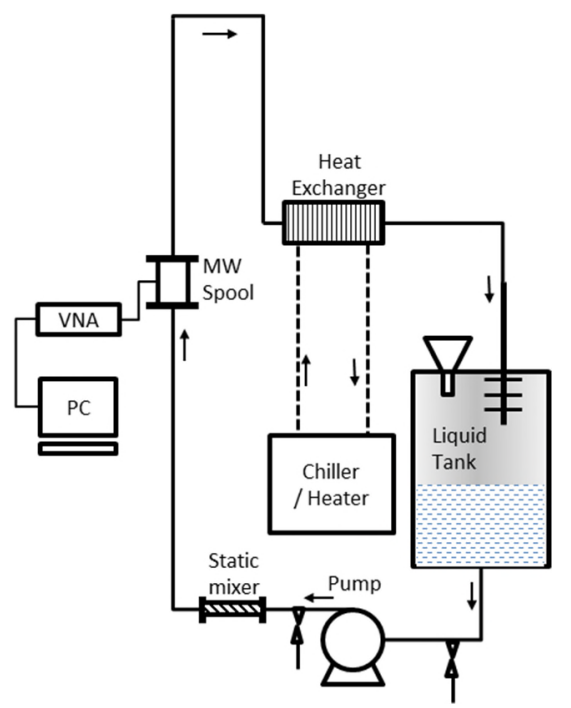

Figure 23: Schematic diagram of the experimental setup

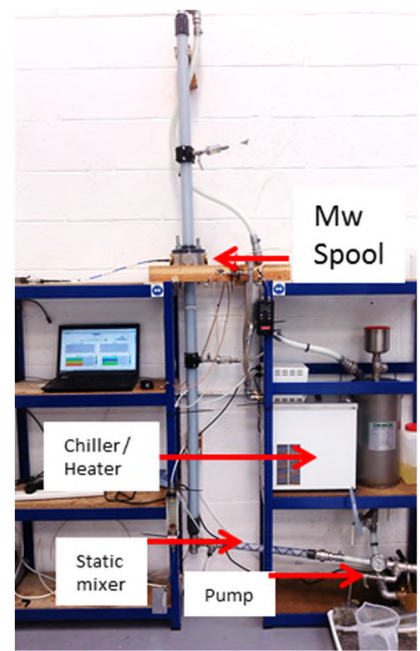

Figure 24: Experimental setup for sensor response measurement

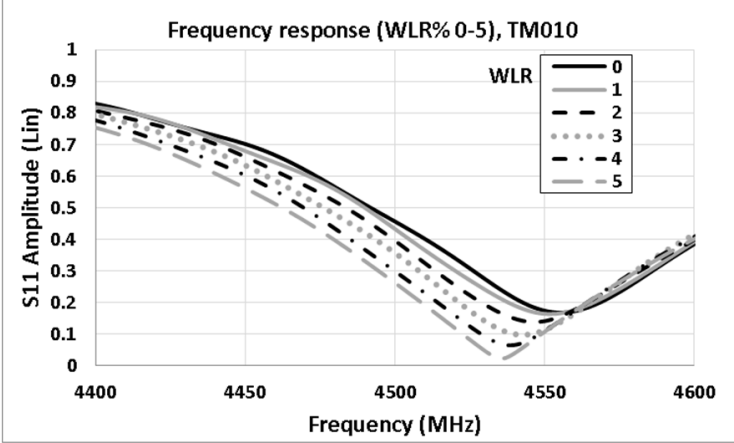

Figure 25: Experimental response of TM010 mode 


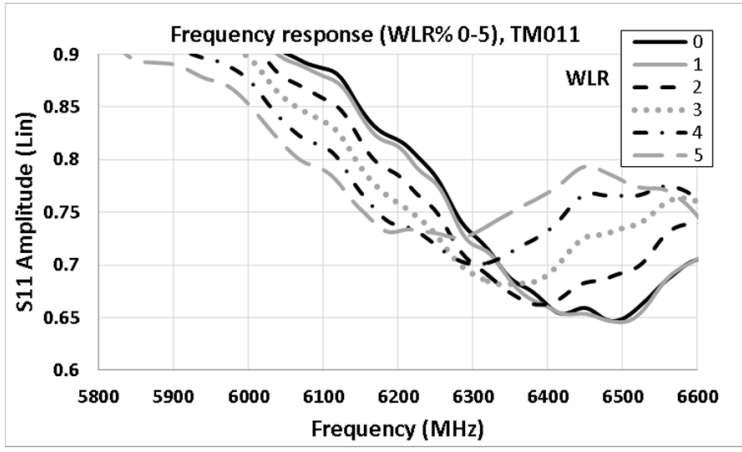

Figure 26: Experimental response of TM011 mode

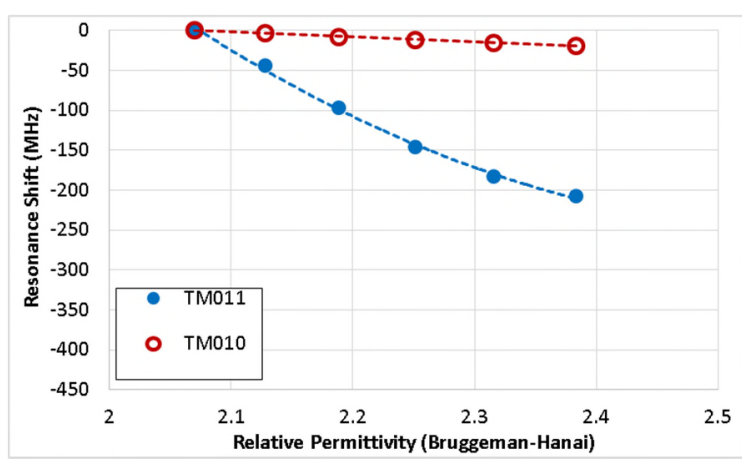

Figure 27: Experimental response sensitivity comparison of TM010 and TM011 modes in Oil-Water flowing mixtures

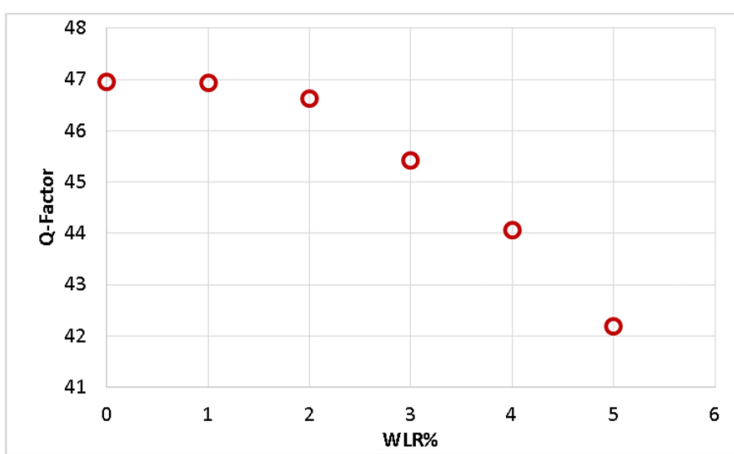

Figure 28: Effect of WLR on Q-Factor of TM010 resonance curve for Oil-Water flowing mixtures

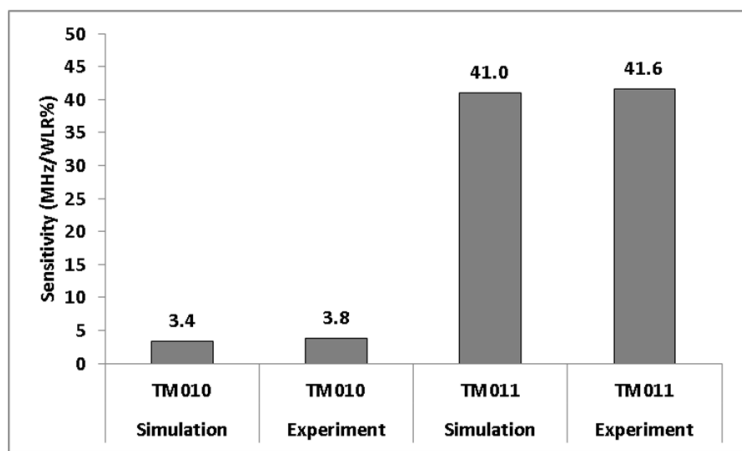

Figure 29: Comparison of simulation and experimental sensitivity (MHz/WLR\%) for modes TM010 and TM011 


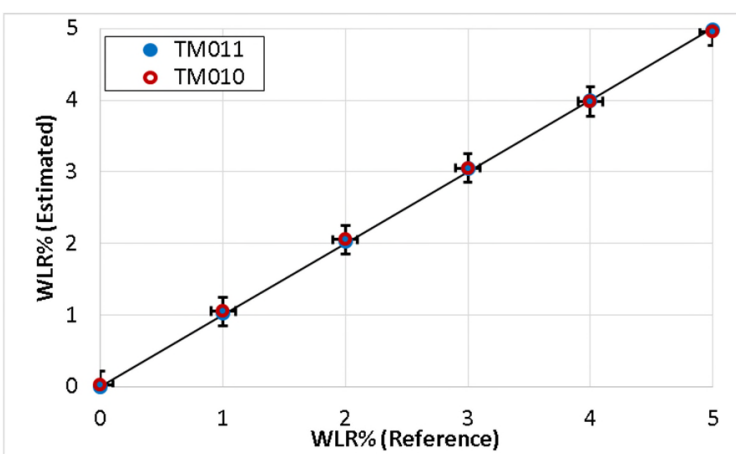

Figure 30: Experimentally estimated WLR\% vs reference WLR\%

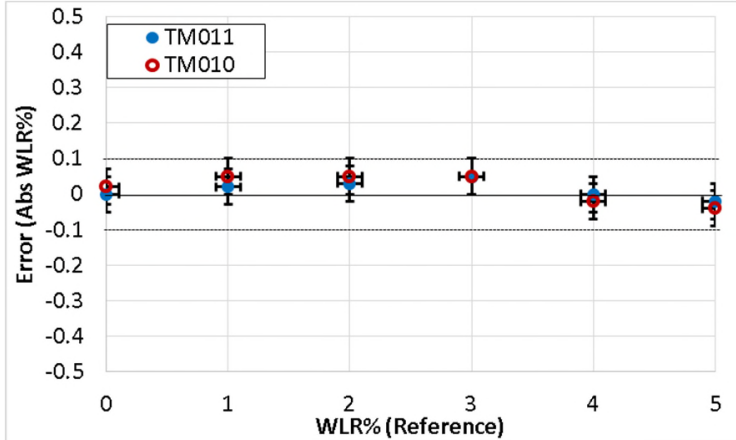

Figure 31: Experimentally estimated error in absolute WLR\% 